\title{
MEASUREMENT OF FLOOR CONVECTORS AT SPECIAL LABORATORY AND FIRST RESULTS
}

\author{
Pavel PEUKERT', Miloš MÜLLER•
}

\begin{abstract}
This article describes the laboratory for measurement of floor convectors in cooling and heating regime. The experimental setup used for the measurement is described. The heating and cooling power are calculated based on the mass flow and temperature measurement. Particle trace method is used for the visualization of the flow inside and outside the convector. A testing measurement is performed to evaluate the influence of the convector covering on the output power.
\end{abstract}

\section{INTRODUCTION}

The floor convectors are devices used for the heating and the cooling in places where the conventional radiators cannot be placed. The convectors utilize the natural convection principle or the forced convection principle. The main objective of the research of the convectors if focused on the increasing their thermal efficiency. As the convectors has been studied for many years there is a wide theoretical background given e.g. in [1], [2]. In general there is no standard for the measurement of the output power of the floor convectors. However the standard ČSN EN 442 [3] can be used for the measurement of the heating power. According the this standard the heating power of the floor convectors can be measured by two methods. The first method is based on the measurement of the mass flow rate through the convector. The second method is based on the measurement of the electric power used for the heating of the fluid. In both cases a given temperature difference must be achieved on the convector vie flow rate regulation.

Our work is focused on the improvement of the heat transfer rates in the convector via optimization of the geometric construction and investigation of the flow inside and outside the convector.

\section{EXPERIMENT AND MEASUREMENTS}

The measurement of the heating and cooling power in the designed laboratory is based on calorimetric equation in form

$$
\dot{Q}=\dot{m} \cdot c \cdot \Delta t=\rho \cdot \dot{V} \cdot c \cdot \Delta t,
$$

where $\dot{Q}$ is power in watts, $\dot{m}$ is the mass flow rate and the $\Delta t$ is temperature difference between the input and the output flow. According to the equation (1) the power of the convector is calculated from the measured volume flux and the measured temperature

\footnotetext{
- Pavel Peukert, Faculty of Mechanical Engineering, Technical University of Liberec, Studentska 2, 46117, Liberec 1, Czech Republic, pavel.peukert@tul.cz

Milos Muller, Faculty of Mechanical Engineering, Technical University of Liberec, Studentska 2, 46117, Liberec 1, Czech Republic, milos.muller@tul.cz
}

This is an Open Access article distributed under the terms of the Creative Commons Attribution License 2.0, which permits unrestricted use, distribution, and reproduction in any medium, provided the original work is properly cited. 
difference. For the normalized power measurement the given temperature difference must be achieved vie volume flux adjustment.

Experimental setup used for the measurement is shown in Figure 1 . For the measurement of the heating power the convector (5) is connected to the electrically heated boiler (2). In order to achieve a stable temperature output the boiler is supplied

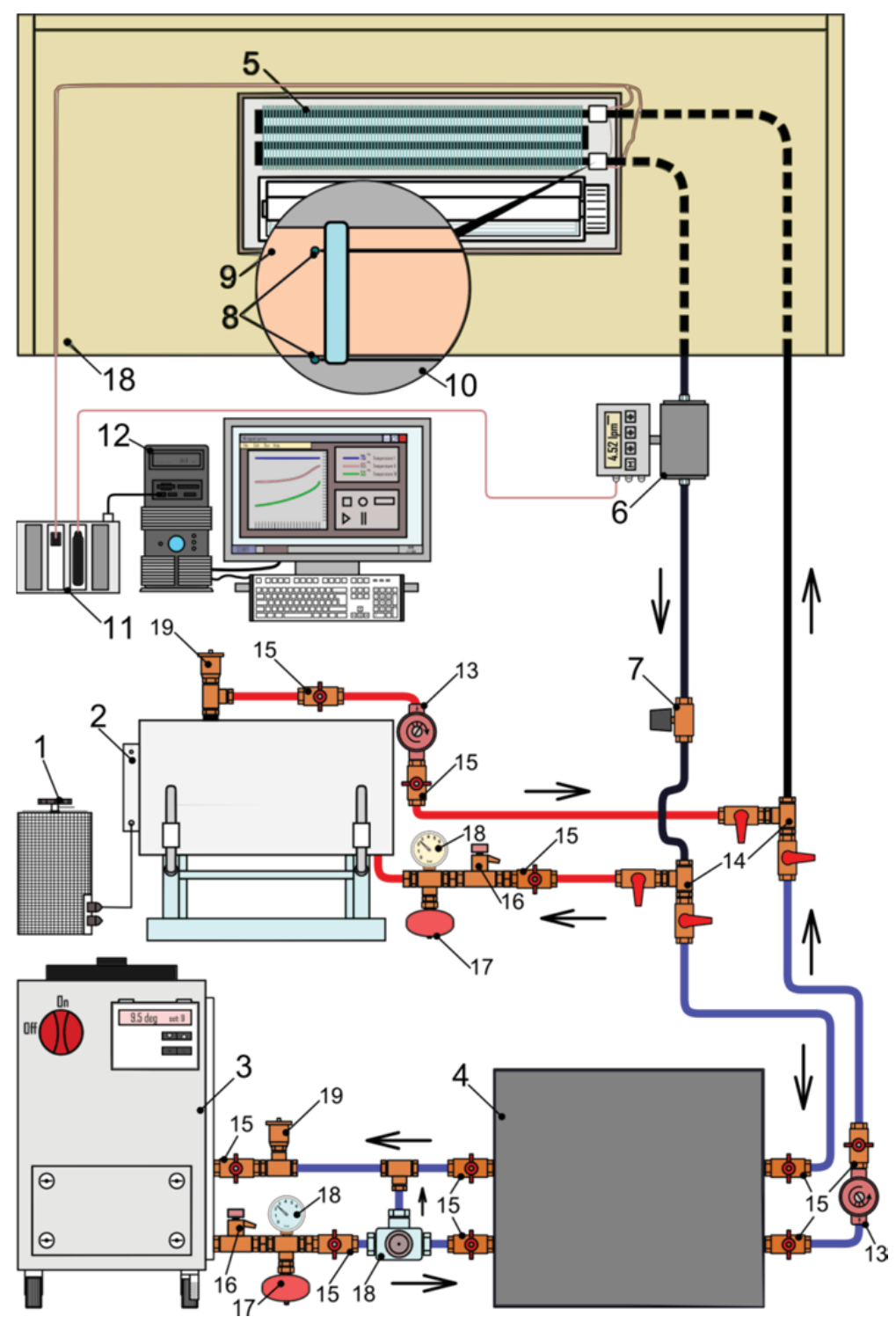

Figure 1: - Laboratory Layout: 1 -autotransformer, 2 -water heater, 3 -industrial chiller, 4 -cold-water mixing device, 5 -complete floor convector, 6 -induction flow meter, 7 -regulation valve, 8 -Thermocouples, 9 -inlet/outlet tube to the convector, 10 - isolation, 11 -docking station with $A / D$ and Thermocouple modules, $12-P C$, 13 - circulation pumps, 14 - valves for opening/closing to the water heating/cooling part, 15 -closing valves, 16 -safety valves, 17 -expansion tanks, 18 - gauges, 19 - deaerator

by the autotransformer (1). This allows to regulate the electric input power of the boiler according to the output heating power of the convector. 
In cooling regime the convector is connected to the cool water reservoir (4), which is supplied by chillier (3). As the chillier has only switch of/on regulation, the cool water reservoir has separate circuit and is heated by separate electric heater regulated via autotransformer.

The temperature difference on the inlet and the outlet is measured via three thermocouples (each). Two thermocouples in each group are used for the measurement.

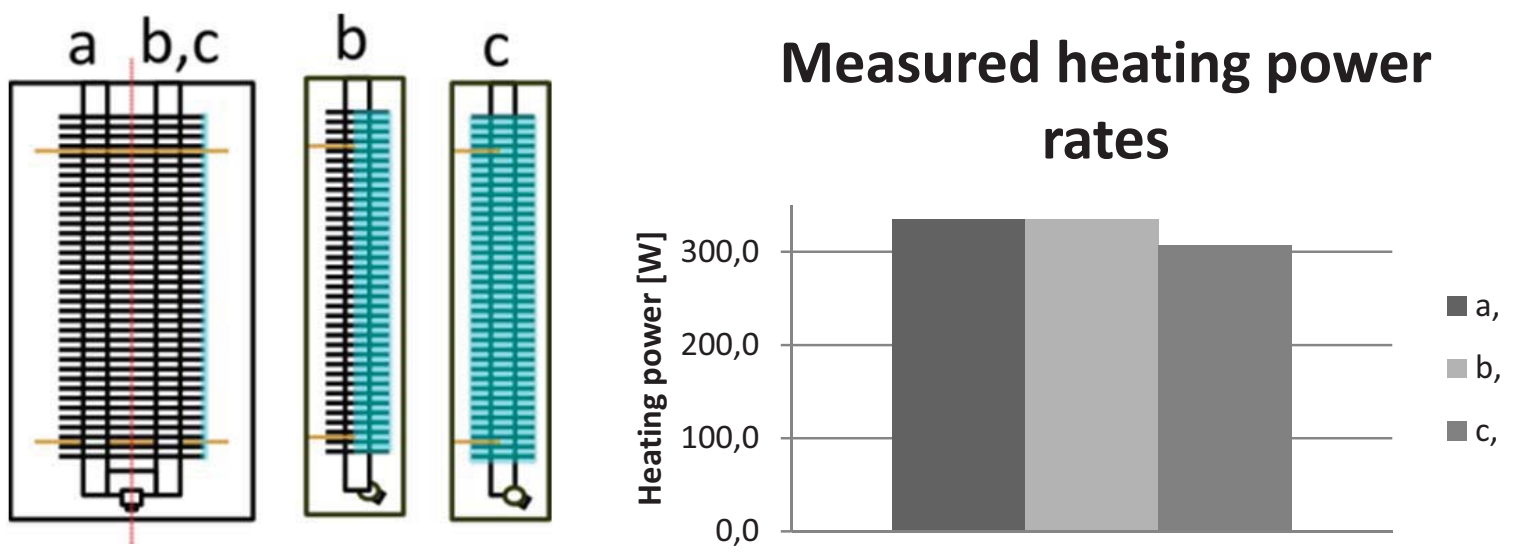

Figure 2: Test arrangement for testing of the effect of side elements (left) and results of measurement (right)

The taken measured value of is that closer to the third thermocouple value. The mass flow is measured using induction flow meter (6) with error under $1.25 \%$ in range from 0.1 to $10 \mathrm{l} / \mathrm{min}$. The thermocouples and the flow meter are connected to the computer vie CompactRio system and monitored in real time with LabView software.

For the visualization of the flow inside and outside the convector a smoke generator is used. The investigated area is illuminated by continuous-wave laser with wavelength 532 $\mathrm{nm}$. The circular laser beam is transformed via cylindrical lens to a sheet of light.

\section{RESULTS}

The goal of the measurement was to show the influence of the side elements on the power of the convector. The elements should improve the flow pattern inside the convector exchanger. The side elements of the full and half size of the convector

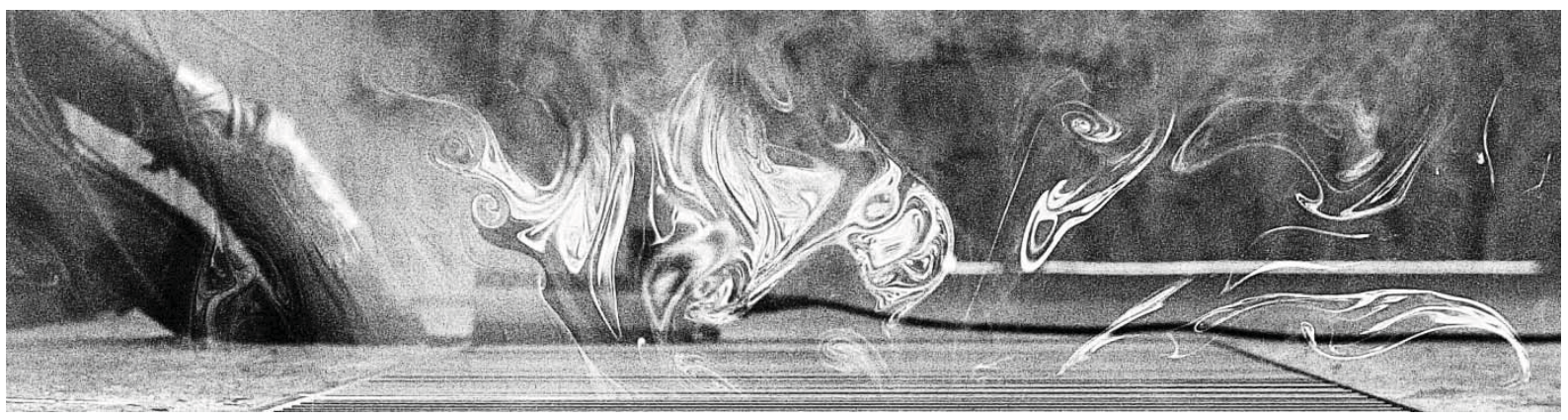

Figure 3: The visualization of the flow on the convector output 
exchanger ribs high were tested. The measured samples are shown on Figure 2. (left). The results of the measurement are shown in Figure 2. (right). It could be seen, that the elements of the size of the half of the ribs high (case b) have no significant effect on the heating power. The elements of the size of the ribs high have rather negative effect on the heating power.

The results of the flow visualization on the convector inlet are shown in the Figure 3. It shows negative effect of the outlet flow suction in the inlet flow, which decrease the thermal efficiency of the convector.

\section{Conclusions}

A new laboratory for measurements of heating and cooling performance of the floor convectors was designed. First result of measurements was already obtained.

\section{ACKNOWLEDGEMENT}

The authors wish to thank the Technology agency of the Czech Republic providing financial support for grant no. TA01020231.

\section{REFERENCES}

[1] Kuppan, T.: Heat Exchanger Design Handbook (1st Edition ed.), CRC Press. IS, 2000

[2] Shah, R., K. and Sekulic, D., P.: Fundamentals of Heat Exchanger Design, John Wiley, New York, 2003

[3] CSN EN 442. Radiators and convectors - Part 2: Test methods and rating, Czech Normalization Institute, Prague, 1998 